\title{
A MULTIDIMENSIONAL WIENER-WINTNER THEOREM AND SPECTRUM ESTIMATION
}

\author{
JOHN J. BENEDETTO
}

ABSTRACT. Sufficient conditions are given for a bounded positive measure $\mu$ on $\mathbb{R}^{d}$ to be the power spectrum of a function $\varphi$. Applications to spectrum estimation are made for the cases in which a signal $\varphi$ is known or its autocorrelation $P_{\phi}$ is known. In the first case, it is shown that

$$
\int|\hat{f}(\gamma)|^{2} d \mu_{\phi}(\gamma)=\lim _{R \rightarrow \infty} \frac{1}{|B(R)|} \int_{B(R)}|f * \varphi(t)|^{2} d t,
$$

where $\widehat{P}_{\varphi}=\mu_{\varphi}, B(R)$ is the $d$-dimensional ball of radius $R$, and $f$ ranges through a prescribed function space. In the second case, an example, which is a variant of the Tomas-Stein restriction theorem, is

$$
\begin{aligned}
\forall f \in & L^{1}\left(\mathbb{R}^{d}\right) \cap L^{p}\left(\mathbb{R}^{d}\right), \\
& \left(\int_{\sum_{d-1}}|\hat{f}(\theta)|^{2} d \mu_{d-1}(\theta)\right)^{1 / 2} \leq\left(\frac{1}{2}\left\|\mu_{d-1}^{\vee}\right\|_{p^{\prime}}^{1 / 2}\right)\left(\|f\|_{1}+\|f\|_{p}\right),
\end{aligned}
$$

where $1 \leq p<2 d /(d+1)$ and the power spectrum $\mu_{d-1}$ is the compactly supported restriction of surface measure to the unit sphere $\sum_{d-1} \subseteq \widehat{\mathbb{R}}^{d}$.

\section{INTRODUCTION}

Wiener's generalized harmonic analysis is a multifaceted subject. It was conceived to deal with noise, hidden periodicities, and turbulence; it was developed in terms of Tauberian theorems and sophisticated spectral analysis; and it continues to play a role in prediction theory, ergodic theory, stochastic processes, and, of course, harmonic analysis [B, W]. An important fact from the general theory is a theorem due to Wiener and Wintner [WW, pp. 241-242]: each bounded positive measure $\mu$ on the real-line $\mathbb{R}$ is the Fourier transform of a function $P$ which can be written in the form,

$$
P(t)=\lim _{R \rightarrow \infty} \frac{1}{2 R} \int_{-R}^{R} \varphi(t+u) \overline{\varphi(u)} d u,
$$

Received by the editors January 30, 1989 and, in revised form, September 11, 1989.

1980 Mathematics Subject Classification (1985 Revision). Primary 42B10.

The author is also Professor of Mathematics at the University of Maryland, College Park.

Research sponsored by the Air Force Office of Scientific Research (AFSC), under Contract F49620-88-C-0028. The United States government is authorized to reproduce and distribute reprints for governmental purposes notwithstanding any copyright notation hereon. 
i.e., each $\mu$ is the power spectrum of some signal $\varphi$ having autocorrelation $P$. In $\S 3$ we extend this result to functions $\varphi$ on $d$-dimensional Euclidean space $\mathbb{R}^{d}$; and we use the theorem as background for proving a $d$-dimensional type of Wiener-Plancherel formula in $\S 5$ and a weighted Fourier transform norm inequality and restriction theorem in $\S 6$. The results in $\S \S 5$ and 6 are viewed in terms of estimating the power spectrum, i.e., "spectrum estimation."

Historically, the Wiener-Wintner theorem (1939) is the desired converse of Wiener's theorem (1930) that the continuous autocorrelation of a signal is the Fourier transform of a bounded positive measure. Since autocorrelations are positive definite this assertion is an easy consequence of Bochner's theorem (1932). It should be pointed out that the Fourier series version of Bochner's theorem was proved by Herglotz in 1911. In any case, in light of examples he constructed, it is natural to presume that Wiener was aware of the possibility of a Wiener-Wintner theorem in the late 1920's, and must have felt sure of it after Khintchine's theorem (1934), cf. Remark 3.4(c). Initially, a major obstacle was to find signals $\varphi$ with continuous singular power spectra; K. Mahler (1927) constructed the first example of such a signal using real power series taking transcendental values for certain algebraic (number) arguments.

$\S 2$ is devoted to notation. Theorem 3.3 (in $\S 3$ ) is our $d$-dimensional version of Wiener and Wintner's theorem. Our formulation is valid for a significant class of positive bounded measures $\mu$ subject only to a mild constraint related to growth at infinity; further, approximants to $\mu$ which arise in our proof are supported by the support of $\mu$. It should be pointed out that Wiener and Wintner's proof is extraordinarily abbreviated, and our proof on $\mathbb{R}^{d}$ is inspired by the intricate argument of Bertrandias for $\mathbb{R}$ [Be], cf. [Ba, Chapitre 2]. $\S 4$ provides a geometrical application of Theorem 3.3 for $\mu_{d-1}$, the restriction of surface measure $\sigma_{d-1}$ to the unit sphere $\Sigma_{d-1}$ of $d$-dimensional Euclidean space $\widehat{\mathbb{R}}^{d}\left(=\mathbb{R}^{d}\right)$.

In Theorem 5.2 (of $\S 5$ ) we derive a formula to express

$$
\int|\hat{f}(\gamma)|^{2} d \mu_{\varphi}(\gamma)
$$

in terms of arithmetic means involving $f$ and a given function $\varphi$. (Integration is over $\widehat{\mathbb{R}}^{d}$ and $\mu_{\varphi}$ is a bounded positive measure on $\widehat{\mathbb{R}}^{d}$ which is the Fourier transform of a $d$-dimensional version of the function $P$ in (1.1); the function $\hat{f}$ denotes the Fourier transform $\hat{f}(\gamma)=\int f(t) e^{-2 \pi i t \cdot \gamma} d t$, where integration is over $\mathbb{R}^{d}$ and $\gamma \in \widehat{\mathbb{R}}^{d}$.) Using Theorem 5.2 with functions $\hat{f}$ having the appropriate shape, we can estimate the mass of $\mu_{\varphi}$ on prescribed regions of $\widehat{\mathbb{R}}^{d}$ in terms of the known functions $f$ and $\varphi$. This is a form of spectrum estimation and further remarks are made in $\S 5$.

The results in $\S 6$ are elementary but of some interest because of recent work on weighted norm inequalities and restriction theorems. The $\left(L^{2}, L^{P}\right)$ restric- 
tion theorem of Tomas and Stein asserts that

$$
\forall f \in L^{1}\left(\mathbb{R}^{d}\right) \cap L^{p}\left(\mathbb{R}^{d}\right), \quad\left(\int_{\Sigma_{d-1}}|\hat{f}(\theta)|^{2} d \sigma_{d-1}(\theta)\right)^{1 / 2} \leq c(p)\|f\|_{p}
$$

for any fixed $1 \leq p \leq 2(d+1) /(d+3)$, e.g., [T]. $L^{p}\left(\mathbb{R}^{d}\right)$ and $\|f\|_{p}$ are the usual Lebesgue space and norm. Discussions of the role of curvature and extensions of (1.2) to other smooth submanifolds are found in [CD, pp. 103-109; S, pp. 325-329]. Weighted $L^{p}$ versions of (1.2) are found in [BH]. Proposition 6.1 is a $\mu$-weighted Fourier transform norm inequality with explicit norm constant in terms of the inverse Fourier transform $P$ of $\mu$; and Proposition 6.2 is a corollary which can be viewed as a restriction result since we take $\mu=\mu_{d-1}$. The inequality (1.2) is deep and Proposition 6.2 is elementary. In (1.2) the constant $c(p)$ is not explicit, $p=2(d+1) /(d+3)$ is largest possible, and the right-hand norm is $\|f\|_{p}$. In Proposition 6.2 the constant is explicit and the values of $p$ extend beyond $2(d+1) /(d+3)$, but the right-hand norm is $\|f\|_{1}+\|f\|_{p}$. As far as spectrum estimation is concerned, Proposition 6.2 can be used to give an upper bound of the mass of $\mu_{d-1}$ on prescribed regions of $\widehat{\mathbb{R}}^{d}$ in terms of the known functions $f$ and $P$. This, too, is a form of spectrum estimation and further remarks are made in $\S 6$. Naturally, in light of Theorem 3.3 and the generality of Proposition 6.1, our discussion of $\mu_{d-1}$ extends to many other measures.

\section{Notation}

Besides the $L^{p}$-spaces, $1 \leq p<\infty$, mentioned in $\S 1$, we shall deal with a number of other spaces. To introduce them we let $\widehat{\mathbb{R}}^{d}\left(=\mathbb{R}^{d}\right)$ be the dual group of $d$-dimensional euclidean space $\mathbb{R}^{d}$ and let $X$ be a locally compact subspace of $\widehat{\mathbb{R}}^{d}$. Then $C_{c}(X)$ is the vector space of complex-valued continuous functions $f: X \rightarrow \mathbb{C}$ having compact support supp $f \subseteq X$. A measure $\mu$ on $X$ is a linear functional defined on $C_{c}(X)$ satisfying $\lim _{j \rightarrow \infty}\left\langle\mu, f_{j}\right\rangle=0$ for every sequence $\left\{f_{j}\right\} \subseteq C_{c}(X)$ having the properties that $\lim _{j \rightarrow \infty}\left\|f_{j}\right\|_{\infty}=0$ and supp $f_{j} \subseteq K$, where $K \subseteq X$ is a compact set independent of $j$ and $\|\cdots\|_{\infty}$ is the usual sup norm, e.g., [Bo]. $M(X)$ is the space of measures on $X$ and $M_{+}(X)=\left\{\mu \in M(X):\langle\mu, f\rangle \geq 0\right.$ for all nonnegative $\left.f \in C_{c}(X)\right\}$ is the space of positive measures on $X$. Similarly, $M_{b}(X)$ is the subspace of $M(X)$ having bounded variation, i.e., the above-mentioned convergence criterion on $C_{c}(X)$ is replaced by $\left(C_{c}(X),\|\cdots\|_{\infty}\right)$; and $M_{b+}(X)$ consists of the positive elements of $M_{b}(X)$. The support of $\mu \in M(X)$ is denoted by supp $\mu$. We write $\langle\mu, f\rangle=\int_{X} f(\gamma) d \mu(\gamma)$ and in the case $X=\widehat{\mathbb{R}}^{d}$ we write $\langle\mu, f\rangle=$ $\int f(\gamma) d \mu(\gamma)$. If $\mu \in M_{b}(X)$ then $\mu$ is well defined on $C_{b}(X)=\{f: f$ is continuous and bounded on $X\}$.

For $p \in(0, \infty), L_{\text {loc }}^{p}\left(\mathbb{R}^{d}\right)$ is the set of functions $f: \mathbb{R}^{d} \rightarrow \mathbb{C}$ for which $|f|^{p}$ is locally integrable with respect to Lebesgue measure. If $\mu \in M_{+}\left(\widehat{\mathbb{R}}^{d}\right)$ 
then $L_{\mu}^{p}\left(\widehat{\mathbb{R}}^{d}\right)$ designates the set of Borel measurable functions $f$ defined $\mu$ a.e. on $\widehat{\mathbb{R}}^{d}$ for which $\|f\|_{p, \mu}=\left(\int|f(\gamma)|^{p} d \mu(\gamma)\right)^{1 / p}<\infty$. There is the standard adjustment for $p=\infty$. Also, we write $p^{\prime}=p /(p-1)$.

The Fourier transform defined in $\S 1$ extends from $L^{1}\left(\mathbb{R}^{d}\right)$ to $M_{b}\left(\mathbb{R}^{d}\right)$ (and beyond); and $\mu^{\vee}$ is the inverse Fourier transform of $\mu \in M_{b}\left(\widehat{\mathbb{R}}^{d}\right)$.

The characteristic function of the set $S \subseteq \mathbb{R}^{d}$ is $\chi_{S}$ and the Lebesgue measure of $S$ is denoted by $|S|$. We set $B(R)=\left\{x \in \mathbb{R}^{d}:|x| \leq R\right\}$ so that $|B(R)|=\omega_{d-1} R^{d} / d$ where $\omega_{d-1}=2 \pi^{d / 2} /(d \Gamma(d / 2))$ is the surface area of $\Sigma_{d-1}$. Finally, we mention that we shall frequently deal with sets of the form $B(R) \backslash B(r)$, and that the boundaries of such sets are unimportant for our results. As such, on this point, we shall not usually be concerned about boundaries of such sets in our calculations.

\section{WIENER-WINTNER THEOREM ON $\mathbb{R}^{d}$}

Given $\mu \in M_{b+}\left(\widehat{\mathbb{R}}^{d}\right)$ and let $\delta_{\omega}$ be the Dirac measure supported by $\{\omega\}$. It is well known that there is a sequence $\left\{\mu_{n}\right\} \subseteq M_{b+}(\operatorname{supp} \mu)$ of positive discrete measures,

$$
\mu_{n}=\sum_{j=1}^{N_{n}} a_{j, n} \delta_{\omega_{j, n}}, \quad a_{j, n}>0,
$$

such that $\left\{\omega_{j, n}: j=1, \ldots, N_{n}\right\} \subseteq \operatorname{supp} \mu$ for each $n$,

$$
\lim _{n \rightarrow \infty}\left\langle\mu_{n}, 1\right\rangle=\langle\mu, 1\rangle,
$$

and $\lim _{n \rightarrow \infty} \mu_{n}=\mu$ in the (vague) topology $\sigma\left(M_{b}\left(\widehat{\mathbb{R}}^{d}\right), C_{c}\left(\widehat{\mathbb{R}}^{d}\right)\right)$, e.g., [Bo, Chapitre III, §2, no. 4]. Actually, (3.1) and the $\sigma\left(M_{b}\left(\widehat{\mathbb{R}}^{d}\right), C_{c}\left(\widehat{\mathbb{R}}^{d}\right)\right)$ convergence allow us to conclude that $\lim _{n \rightarrow \infty} \mu_{n}=\mu$ in the "Levy" topology $\sigma\left(M_{b}\left(\widehat{\mathbb{R}}^{d}\right), C_{b}\left(\widehat{\mathbb{R}}^{d}\right)\right)$, e.g., [Ma, pp. 91-93]. Thus, we have

$$
\forall f \in C_{b}\left(\widehat{\mathbb{R}}^{d}\right), \quad \lim _{n \rightarrow \infty} \int f(\gamma) d \mu_{n}(\gamma)=\int f(\gamma) d \mu(\gamma) .
$$

For a given $\mu \in M_{b+}\left(\widehat{\mathbb{R}}^{d}\right)$ and sequence $\left\{\mu_{n}\right\} \subseteq M_{b+}(\operatorname{supp} \mu)$ as above, we define

$$
\varphi_{n}(t)=\sum_{j=1}^{N_{n}} a_{j, n}^{1 / 2} e^{2 \pi i t \cdot \omega_{j, n}}
$$

so that

$$
\left\|\varphi_{n}\right\|_{\infty} \leq \sum_{j=1}^{N_{n}} a_{j, n}^{1 / 2} \leq N_{n} \sup _{1 \leq j \leq N_{n}} a_{j, n}^{1 / 2} .
$$

The signal $\varphi$ used in our statement of the Wiener-Wintner theorem (Theorem 3.3) is defined in the paragraph following Lemma 3.2 and depends on these partial signals $\varphi_{n}$. 
Lemma 3.1. For each $n$,

$$
\lim _{R \rightarrow \infty} \frac{1}{|B(R)|} \int_{B(R)} \varphi_{n}(t+x) \overline{\varphi_{n}(x)} d x=\mu_{n}^{\vee}(t),
$$

uniformly on $\mathbb{R}^{d}$.

Proof. The left-hand side of (3.2) is $\mu_{n}^{\vee}(t)+\lim _{R \rightarrow \infty} \varepsilon(R, t, n)$ (if this limit exists), where

$$
\begin{aligned}
\varepsilon(R, t, n)= & \frac{1}{|B(R)|} \sum_{j \neq k} a_{j, n}^{1 / 2} a_{k, n}^{1 / 2} e^{2 \pi i t \cdot \omega_{j, n}} \int_{B(R)} e^{2 \pi i x \cdot\left(\omega_{j, n}-\omega_{k, n}\right)} d x \\
= & \frac{2 \pi}{|B(R)|} \sum_{j \neq k} a_{j, n}^{1 / 2} a_{k, n}^{1 / 2} e^{2 \pi i t \cdot \omega_{j, n}}\left|\omega_{j, n}-\omega_{k, n}\right|^{-((d-2) / 2)} \\
& \cdot \int_{0}^{R} r^{d / 2} J_{(d-2) / 2}\left(2 \pi r\left|\omega_{j, n}-\omega_{k, n}\right|\right) d r
\end{aligned}
$$

and $J_{(d-2) / 2}$ is the Bessel function of order $(d-2) / 2$. Since $\left|J_{(d-2) / 2}(s)\right| \leq 1$ (at the origin) and $J_{(d-2) / 2}(s)=O\left(s^{-1 / 2}\right), s \rightarrow \infty$, we see that $\lim _{R \rightarrow \infty} \varepsilon(R, t, n)$ $=0$ independently of $t$. Q.E.D.

We shall also need the following easy fact.

Lemma 3.2. Given a sequence $\left\{R_{n}\right\}$ increasing to infinity and $\varphi \in L_{\text {loc }}^{\infty}\left(\mathbb{R}^{d}\right)$, fix $t \in \mathbb{R}^{d}$. Then for each fixed $p$,

$$
\lim _{R \rightarrow \infty} \frac{1}{|B(R)|} \int_{B\left(R_{p}\right)} \varphi(t+x) \overline{\varphi(x)} d x=0 .
$$

We use Lemma 3.1 to define a specific sequence $\left\{R_{n}\right\}$ and a specific function $\varphi$ in the following way. From the uniform convergence we know that

$$
\begin{gathered}
\forall n \geq 1, \quad \exists A_{n} \geq A_{n-1} \text { such that } \forall t \in \mathbb{R}^{d} \text { and } \forall R \geq A_{n}, \\
\left|\frac{1}{|B(R)|} \int_{B(R)} \varphi_{n}(t+x) \overline{\varphi_{n}(x)} d x-\mu_{n}^{\vee}(t)\right|<\frac{1}{2^{n+1}} .
\end{gathered}
$$

We set $R_{n}=\left(A_{1}+1\right)\left(A_{2}+2\right) \cdots\left(A_{n}+n\right)$ so that each $R_{n} \geq n$ ! and the sequences $\left\{R_{n}\right\},\left\{R_{n+1} / R_{n}\right\}=\left\{A_{n+1}+n+1\right\}$, and $\left\{R_{n+1}-R_{n}\right\}=\left\{\left(A_{1}+1\right) \cdots\right.$ $\left.\left(A_{n}+n\right)\left(A_{n+1}+n\right)\right\}$ increase to infinity. For this sequence $\left\{R_{n}\right\}$ and for $\left\{\varphi_{n}\right\}$ defined above we define $\varphi$ on $\mathbb{R}^{d}$ be setting $\varphi(t)=\varphi_{n}(t)$ for $R_{n}<|t|<R_{n+1}$, $n \geq 1$, and letting $\varphi(t)=0$ for $|t|<R_{1}$. Clearly, $\varphi \in L_{\text {loc }}^{\infty}\left(\mathbb{R}^{d}\right)$ and the values of $\varphi(t)$ for $|t|=r_{n}$ are not important.

Using this notation, setup, and the lemmas, we can state the Wiener-Wintner construction on $\mathbb{R}^{d}$.

Theorem 3.3. Given $\mu \in M_{b+}\left(\widehat{\mathbb{R}}^{d}\right)$ with corresponding functions $\left\{\varphi_{n}\right\}$ and $\varphi \in$ $L_{\mathrm{loc}}^{\infty}\left(\mathbb{R}^{d}\right)$. Assume

$$
\lim _{n \rightarrow \infty} \frac{1}{n !} \sum_{j=2}^{n} \max \left(\left\|\varphi_{j-1}\right\|_{\infty},\left\|\varphi_{j}\right\|_{\infty}\right) \max \left(\left\|\varphi_{j}\right\|_{\infty},\left\|\varphi_{j+1}\right\|_{\infty}\right)=0
$$


Then, for each $t \in \mathbb{R}^{d}$,

$$
\lim _{R \rightarrow \infty} \frac{1}{|B(R)|} \int_{B(R)} \varphi(t+x) \overline{\varphi(x)} d x=\mu^{\vee}(t) .
$$

Proof. (a) Given $t \in \mathbb{R}^{d}$ and $\varepsilon>0$. Because of our setup we can choose $p_{1}=p_{1}(t)$ for which $R_{k+1}-|t|>R_{k}+|t|$ for all $k \geq p_{1}$ and we can choose $p_{2}=p_{2}(\varepsilon, t)>p_{1}$ such that

$$
\forall k \geq p_{2}, \quad\left|\mu_{k}^{\vee}(t)-\mu^{\vee}(t)\right|<\varepsilon .
$$

We shall find $p=p(\varepsilon, t)>p_{2}$ such that for all sufficiently large $R$,

$$
\left|\frac{1}{|B(R)|} \int_{B(R) \backslash B\left(R_{p}\right)} \varphi(t+x) \overline{\varphi(x)} d x-\mu^{\vee}(t)\right|<14 \varepsilon ;
$$

and, hence, (3.5) is a consequence of Lemma 3.2 and a “ $\varlimsup$ lim " argument.

(b) For each $p>p_{2}$ and each $R>R_{p}$, write

$$
\begin{aligned}
& \frac{1}{|B(R)|} \int_{B(R) \backslash B\left(R_{p}\right)} \varphi(t+x) \overline{\varphi(x)} d x=\sum_{k=p}^{n-1}(b(k, R)+c(k, R)) \\
& \quad+\frac{1}{|B(R)|} \int_{B(R) \backslash B\left(R_{n}\right)} \varphi(t+x) \overline{\varphi(x)} d x,
\end{aligned}
$$

where

$$
\begin{aligned}
& b(k, R)=\frac{1}{|B(R)|} \int_{B\left(R_{k+1}-|t|\right) \backslash B\left(R_{k}\right)} \varphi(t+x) \overline{\varphi(x)} d x, \\
& c(k, R)=\frac{1}{|B(R)|} \int_{B\left(R_{k+1}\right) \backslash B\left(R_{k+1}-|t|\right)} \varphi(t+x) \overline{\varphi(x)} d x,
\end{aligned}
$$

and $n=n(R)$ is the largest integer $n$ for which $R_{n} \leq R$.

In part (c) we shall verify that for $p>p_{1}$

$$
\lim _{R \rightarrow \infty} \sum_{k=p}^{n-1} c(k, R)=0
$$

Parts $(\mathrm{d})-(\mathrm{h})$ are devoted to showing that there are $p_{3}=p_{3}(\varepsilon, t)>p_{2}$ and $R(\varepsilon, t)>p_{3}$ such that, for $R(\varepsilon, t)>p \geq p_{3}$,

$$
\begin{aligned}
\forall R> & R(\varepsilon, t), \quad \sum_{k=p}^{n-1} b(k, R) \\
& =\mu^{\vee}(t) \frac{1}{|B(R)|} \sum_{k=p}^{n-1}\left(\left|B\left(R_{k+1}-|t|\right)\right|-\left|B\left(R_{k}\right)\right|\right)+r(p, R, t),
\end{aligned}
$$

where $|r(p, R, t)|<7 \varepsilon+1 / 2^{p-1}$. Parts (i)-(k) contain the proof that

$$
\begin{aligned}
\forall R>R(\varepsilon, t), \quad \frac{1}{|B(R)|} & \int_{B(R) \backslash B\left(R_{n}\right)} \varphi(t+x) \overline{\varphi(x)} d x \\
& =\mu^{\vee}(t)\left(1-\frac{\left|B\left(R_{n}\right)\right|}{|B(R)|}\right)+s(R, t),
\end{aligned}
$$


where $|s(R, t)|<4 \varepsilon+1 / 2^{n}$. (3.7) is obtained in part (1) by combining (3.9), (3.10), and (3.11).

(c) If $x \in B\left(R_{k+1}\right) \backslash B\left(R_{k+1}-|t|\right)$ then $R_{k} \leq|x+t| \leq R_{k+2}$. Thus, setting $c_{k}=\left\|\varphi_{k}\right\|_{\infty} \max \left(\left\|\varphi_{k}\right\|_{\infty},\left\|\varphi_{k+1}\right\|_{\infty}\right)$, we have

$$
\begin{aligned}
\sum_{k=p}^{n-1} c(k, R) & \leq \frac{1}{R^{d}} \sum_{k=p}^{n-1} c_{k}\left[R_{k+1}^{d}-\left(R_{k+1}-|t|\right)^{d}\right] \\
& \leq \frac{1}{R_{n}^{d}} \sum_{k=p}^{n-1} c_{k} R_{k+1}^{d}\left[1-\left(1-\frac{|t|}{R_{k+1}}\right)^{d}\right] \\
& \leq \frac{1}{R_{n}^{d}} \sum_{k=p}^{n-1} c_{k} R_{k+1}^{d} \sum_{j=1}^{d}\left(\begin{array}{l}
d \\
j
\end{array}\right)\left(\frac{|t|}{R_{k+1}}\right)^{j} \\
& \leq\left(\frac{1+|t|}{R_{n}}\right)^{d} \sum_{k=p}^{n-1} c_{k} R_{k+1}^{d-1} \leq \frac{(1+|t|)^{d}}{n !} \sum_{k=1}^{n-1} c_{k} .
\end{aligned}
$$

(3.9) follows by comparing the right-hand side of this estimate with our hypothesis (3.4).

(d) In order to estimate $b(k, R)$ we define the sets

$$
A_{k}(t)=\left\{x \in B\left(R_{k+1}-|t|\right) \backslash B\left(R_{k}\right): t+x \in B\left(R_{k+1}\right) \backslash B\left(R_{k}\right)\right\}
$$

and

$$
A_{k-1}(t)=\left\{x \in B\left(R_{k+1}-|t|\right) \backslash B\left(R_{k}\right): t+x \in B\left(R_{k}\right) \backslash B\left(R_{k-1}\right)\right\} .
$$

Then, for $k>p_{1}, B\left(R_{k+1}-|t|\right) \backslash B\left(R_{k}\right)=A_{k}(t) \cup A_{k-1}(t)$, a disjoint union; and we have

$$
\begin{aligned}
b(k, R)= & \frac{1}{|B(R)|} \int_{A_{k}(t) \cup B\left(R_{k}\right)} \varphi_{k}(t+x) \overline{\varphi_{k}(x)} d x \\
& -\frac{1}{|B(R)|} \int_{B\left(R_{k}\right)} \varphi_{k}(t+x) \overline{\varphi_{k}(x)} d x \\
& +\frac{1}{|B(R)|} \int_{A_{k-1}(t)} \varphi_{k-1}(t+x) \overline{\varphi_{k}(x)} d x .
\end{aligned}
$$

We shall estimate these three integrals. The first two will involve the factor $\mu^{\vee}(t)$ and the latter becomes small by the size of $A_{k-1}(t)$.

In the process of making these estimates we need the bound,

$$
\frac{1}{R_{n}^{d}} \sum_{k=1}^{n} R_{k}^{d} \leq 2 .
$$

In fact, the left-hand side of (3.13) is bounded by

$$
1+\frac{1}{n}+\frac{1}{n(n-1)}+\cdots+\frac{1}{n !},
$$


and it is easy to see that for each $k \geq 3$

$$
\frac{1}{(k+1) !} \sum_{j=0}^{k+1} j !<\frac{1}{k !} \sum_{j=0}^{k} j ! \leq \frac{5}{3}
$$

hence, (3.13) is valid.

(e) Our initial step in estimating the first integral on the right-hand side of (3.12) is to prove that there is $p_{3}=p_{3}(\varepsilon, t)>p_{2}$ such that for all $k \geq p_{3}$,

$$
\left|\frac{1}{\left|B\left(R_{k+1}-|t|\right)\right|}\left(\int_{B\left(R_{k+1}-|t|\right)} \varphi_{k}(t+x) \overline{\varphi_{k}(x)} d x-\int_{A_{k}(t) \cup B\left(R_{k}\right)} \varphi_{k}(t+x) \overline{\varphi_{k}(x)} d x\right)\right|<\varepsilon .
$$

The difference of the two integrals is the integral over $A_{k-1}(t)$, and if $x \in$ $A_{k-1}(t)$ then $R_{k} \leq|x| \leq R_{k}+|t|$. Thus, setting $d_{k}=\left\|\varphi_{k}\right\|_{\infty}^{2}$, the left-hand side of (3.14) is bounded by

$$
\frac{d_{k}}{\left(R_{k+1}-|t|\right)^{d}}\left[\left(R_{k}+|t|\right)^{d}-R_{k}^{d}\right] \leq \frac{d d_{k}}{\left(R_{k+1}-|t|\right)^{d}}\left(R_{k}+|t|\right)^{d-1},
$$

where this last inequality is a consequence of the mean value theorem. By (3.4), the right-hand side of (3.15) tends to 0 as $k \rightarrow \infty$; and (3.14) is obtained.

By means of (3.3), (3.6), (3.14), and the triangle inequality we have

$$
\left|\frac{1}{\left|B\left(R_{k+1}-|t|\right)\right|} \int_{A_{k}(t) \cup B\left(R_{k}\right)} \varphi_{k}(t+x) \overline{\varphi_{k}(x)} d x-\mu^{\vee}(t)\right|<2 \varepsilon+\frac{1}{2^{k+1}},
$$

where $k \geq p_{3}$. Clearly, we can write (3.16) in the form,

$$
\begin{gathered}
\frac{1}{\left|B\left(R_{k+1}-|t|\right)\right|} \int_{A_{k}(t) \cup B\left(R_{k}\right)} \varphi_{k}(t+x) \overline{\varphi_{k}(x)} d x \\
=\mu^{\vee}(t)+\beta_{k}(t)\left(2 \varepsilon+\frac{1}{2^{k+1}}\right),
\end{gathered}
$$

where $\left|\beta_{k}(t)\right|<1$ and $k \geq p_{3}$.

(f) To estimate the second integral on the right-hand side of (3.12) we use (3.3), (3.6), and the triangle inequality to write

$$
\left|\frac{1}{B\left(R_{k}\right)} \int_{B\left(R_{k}\right)} \varphi_{k}(t+x) \overline{\varphi_{k}(x)} d x-\mu^{\vee}(t)\right|<\varepsilon+\frac{1}{2^{k+1}},
$$

for $k>p_{2}$. Analogous to part (e), (3.18) yields

$$
\frac{1}{\left|B\left(R_{k}\right)\right|} \int_{B\left(R_{k}\right)} \varphi_{k}(t+x) \overline{\varphi_{k}(x)} d x=\mu^{\vee}(t)+\gamma_{k}(t)\left(\varepsilon+\frac{1}{2^{k+1}}\right),
$$

where $\left|\gamma_{k}(t)\right|<1$ and $k>p_{2}$.

(g) Since $A_{k-1}(t) \subseteq B\left(R_{k}+|t|\right) \backslash\left(R_{k}\right)$, the third integral on the right-hand side of (3.12) is bounded by

$$
\begin{aligned}
& \frac{1}{\left|B\left(R_{k}\right)\right|} \int_{A_{k-1}(t)}\left|\varphi_{k-1}(t+x) \overline{\varphi_{k}(x)}\right| d x \\
& \quad \leq \frac{\left\|\varphi_{k-1}\right\|_{\infty}\left\|\varphi_{k}\right\|_{\infty}}{R_{n}^{d}}\left[\left(R_{k}+|t|\right)^{d}-R_{k}^{d}\right] .
\end{aligned}
$$


(h) By parts (e), (f), and (g) we can write

$$
\begin{aligned}
\sum_{k=p}^{n-1} b(k, R)= & \frac{1}{|B(R)|} \sum_{k=p}^{n-1}\left|B\left(R_{k+1}-|t|\right)\right|\left(\mu^{\vee}(t)+\beta_{k}(t)\left(2 \varepsilon+\frac{1}{2^{k+1}}\right)\right) \\
& -\frac{1}{|B(R)|} \sum_{k=p}^{n-1}\left(\left|B\left(R_{k}\right)\right|\right)\left(\mu^{\vee}(t)+\gamma_{k}(t)\left(\varepsilon+\frac{1}{2^{k+1}}\right)\right) \\
& +\frac{1}{|B(R)|} \sum_{k=p}^{n-1} \int_{A_{k-1}(t)} \varphi_{k-1}(t+x) \overline{\varphi_{k}(x)} d x .
\end{aligned}
$$

To estimate the last term in (3.21) we use (3.20) and compute

$$
\begin{aligned}
& \left.\frac{1}{R_{n}^{d}} \sum_{k=p}^{n-1}\left\|\varphi_{k-1}\right\|_{\infty}\left\|\varphi_{k}\right\|_{\infty}\left[\left(R_{k}+|t|\right)^{d}-R_{k}^{d}\right)\right] \\
& \leq \frac{d|t|}{R_{n}^{d}} \sum_{k=p}^{n-1}\left\|\varphi_{k-1}\right\|_{\infty}\left\|\varphi_{k}\right\|_{\infty}\left(R_{k}+|t|\right)^{d-1} \\
& \quad \leq \frac{d|t| 2^{d-1}}{R_{n}} \sum_{k=p}^{n-1}\left\|\varphi_{k-1}\right\|_{\infty}\left\|\varphi_{k}\right\|_{\infty}\left(\frac{R_{k}}{R_{n}}\right)^{d-1} .
\end{aligned}
$$

By (3.4), this term is less than $\varepsilon$ for all large $R$. Consequently, (3.21) allows us to obtain (3.10). It is at this point that we invoke (3.13).

(i) We now estimate the last term in (3.8). Analogous to part (d) we define the sets $A_{R, k}(t)=\left\{x \in B(R) \backslash B\left(R_{n}\right): t+x \in B\left(R_{k+1}\right) \backslash B\left(R_{k}\right)\right\}$ for $k=n-1$, $n, n+1$. Then, for $n>p_{1}, B(R) \backslash B\left(R_{n}\right)=A_{R, n-1}(t) \cup A_{R, n}(t) \cup A_{R, n+1}(t)$, a disjoint union. (Actually, we have $n \geq p_{3}$.) Therefore, the last term of (3.8) is

$$
\begin{aligned}
& \frac{1}{|B(R)|} \int_{B(R) \backslash B\left(R_{n}\right)} \varphi(t+x) \overline{\varphi(x)} d x \\
& =\frac{1}{|B(R)|} \int_{A_{R, n}(t) \cup B\left(R_{n}\right)} \varphi_{n}(t+x) \overline{\varphi_{n}(x)} d x, \\
& \quad-\frac{\left|B\left(R_{n}\right)\right|}{|B(R)|}\left(\frac{1}{\left|B\left(R_{n}\right)\right|} \int_{B\left(R_{n}\right)} \varphi_{n}(t+x) \overline{\varphi_{n}(x)} d x\right) \\
& \quad+\frac{1}{|B(R)|}\left(\int_{A_{R, n-1}(t)} \varphi_{n-1}(t+x) \overline{\varphi_{n}(x)} d x\right. \\
& \left.\quad \quad \quad \int_{A_{R, n+1}(t)} \varphi_{n+1}(t+x) \overline{\varphi_{n}(x)} d x\right) .
\end{aligned}
$$

(j) The last term of (3.22) is estimated as in part (g). The inclusions $A_{R, n-1}(t)$ $\subseteq B\left(R_{n}+|t|\right) \backslash B\left(R_{n}\right)$ and $A_{R, n+1}(t) \subseteq B(R) \backslash B\left(R_{n+1}-|t|\right)$ are valid, but it should be observed that $A_{R, n+1}(t)$ can be the empty set, e.g., if $R<R_{n+1}-|t|$. In any 
case, this last term of (3.22) is dominated by

$$
\begin{aligned}
\frac{1}{R^{d}}\{ & \left.\left\|\varphi_{n-1}\right\|_{\infty}\left\|\varphi_{n}\right\|_{\infty}\left[\left(R_{n}+|t|\right)^{d}-R_{n}^{d}\right)\right] \\
& \left.+\left\|\varphi_{n+1}\right\|_{\infty}\left\|\varphi_{n}\right\|_{\infty}\left[R^{d}-\left(R_{n+1}-|t|\right)^{d}\right]\right\}
\end{aligned}
$$

where " $\left[R^{d}-\left(R_{n}-|t|\right)^{d}\right]$ " is defined to be 0 if $R<R_{n+1}-|t|$. Using the mean value theorem again, (3.23) is bounded by

$$
\frac{d|t|}{R_{n}}\left\|\varphi_{n-1}\right\|_{\infty}\left\|\varphi_{n}\right\|_{\infty}\left(\frac{R_{n}+|t|}{R_{n}}\right)^{d-1}+\frac{d\left(R-\left(R_{n+1}-|t|\right)\right)}{R}\left\|\varphi_{n+1}\right\|_{\infty}\left\|\varphi_{n}\right\|_{\infty} .
$$

Our hypothesis (3.4) allows us to conclude that this quantity is less than $\varepsilon$ for all large $R$. (For the second term, recall that $R<R_{n+1}$.)

(k) Our estimation of the first two integrals on the right-hand side of (3.22) follows steps (e) and (f). For example, analogous to (3.14), we have

$$
\begin{aligned}
& \left|\frac{1}{|B(R)|}\left(\int_{B(R)} \varphi_{n}(t+x) \overline{\varphi_{n}(x)} d x-\int_{A_{R, n}(t) \cup B\left(R_{n}\right)} \varphi_{n}(t+x) \overline{\varphi_{n}(x)} d x\right)\right| \\
& \quad \leq \frac{1}{|B(R)|}\left\|\varphi_{n}\right\|_{\infty}^{2}\left(\left|A_{R, n-1}(t)\right|+\left|A_{R, n+1}(t)\right|\right) ;
\end{aligned}
$$

and the right-hand side is less than $\varepsilon$ for all large $R$ by the calculation used in step (j). Proceeding in this way, as in parts (e) and (f), we obtain

$$
\begin{gathered}
\frac{1}{|B(R)|} \int_{A_{R, n}(t) \cup B\left(R_{n}\right)} \varphi_{n}(t+x) \overline{\varphi_{n}(x)} d x \\
=\mu^{\vee}(t)+\beta_{R, n}(t)\left(2 \varepsilon+\frac{1}{2^{n+1}}\right)
\end{gathered}
$$

and

$$
\frac{1}{\left|B\left(R_{n}\right)\right|} \int_{B\left(R_{n}\right)} \varphi_{n}(t+x) \overline{\varphi_{n}(x)} d x=\mu^{\vee}(t)+\gamma_{R, n}(t)\left(\varepsilon+\frac{1}{2^{n+1}}\right),
$$

where $\left|\beta_{R, n}(t)\right|,\left|\gamma_{R, n}(t)\right|<1$. Combining these facts with part (j) and (3.22), we have verified (3.11).

(l) For $p \geq p_{3}$ large enough and for all large $R, n(R)>p$, the right-hand side of (3.8) is

$$
\mu^{\vee}(t)+\frac{\mu^{\vee}(t)}{|B(R)|}\left[\sum_{k=p}^{n-1}\left(\left|B\left(R_{k+1}-|t|\right)\right|-\left|B\left(R_{k}\right)\right|\right)-\left|B\left(R_{n}\right)\right|\right]
$$

plus an error term bounded by $13 \varepsilon$. This is a consequence of $(3.9),(3.10)$, and (3.11). We rewrite the second term of (3.24) as

$$
\begin{aligned}
-\frac{\mu^{\vee}(t)}{|B(R)|}\left[\left(\left|B\left(R_{n}\right)\right|\right.\right. & \left.-\left|B\left(R_{n}-|t|\right)\right|\right)+\left(\left|B\left(R_{n-1}\right)\right|-\left|B\left(R_{n-1}-|t|\right)\right|\right) \\
& \left.+\cdots+\left(\left|B\left(R_{p+1}\right)\right|-\left|B\left(R_{p+1}-|t|\right)\right|\right)+\left|B\left(R_{p}\right)\right|\right],
\end{aligned}
$$


which, in absolute value, is bounded by

$$
\begin{aligned}
& \frac{\left|\mu^{\vee}(t)\right|}{R^{d}}\left[\sum_{k=p}^{n}\left(\left(R_{k}^{d}-\left(R_{k}-|t|\right)^{d}\right)+R_{p}^{d}\right]\right. \\
& \leq\left|\mu^{\vee}(t)\right| \frac{d|t|}{R} \sum_{k=p}^{n-1}\left(\frac{R_{k}}{R}\right)^{d-1}+\left|\mu^{\vee}(t)\right|\left(\frac{R_{p}}{R}\right)^{d},
\end{aligned}
$$

and which, in turn, is less than $\varepsilon$ for all large $R$. Thus (3.7) is verified, and, by the observation in part (a), the theorem is proved. Q.E.D.

Remark 3.4. (a) The hypothesis (3.4) can be weakened. There is left unanswered the problem of characterizing those $\mu$ for which $\varphi \in L^{\infty}\left(\mathbb{R}^{d}\right)$.

(b) Our proof of Theorem 3.3 is almost, but not entirely, a "time-domain" analysis. Since autocorrelations are frequently the measurable or observable quantity in an experiment, e.g. the Michelson interfeormeter, it is natural to ask if there is an intrinsic method of constructing a solution $\varphi$ of (1.1) for a given positive definite function $P$. By "intrinsic" we mean that it is not required to pass to the power spectrum $\mu=\widehat{P}$ in order to define segments $\varphi_{n}$ of the signal in terms of square roots and approximants to $\mu$. Such a method would not only supercede our approach but would be invaluable in applications.

(c) Wiener was well aware of the statistical ramifications of his generalized harmonic analysis even though his original formulation was made in terms of specific sample functions. In this regard we point out the following statistical perspective on Theorem 3.3. From the program begun by Khintchine (1934) we know that for any continuous positive definite function $P$ there is a strictly stationary Gaussian stochastic process $x$ whose covariance is $P$. Maruyama's theorem (1949) asserts that this process is ergodic if $\widehat{P}$ is a continuous measure. Consequently, for continuous power spectra, Birkhoff's ergodic theorem provides sample functions $\varphi$ (of the process $x$ ) which are solutions of (1.1). This nonconstructive analysis can be extended to $\mathbb{R}^{d}$ at the expense of verifying Maruyama's theorem for random fields and of implementing Wiener's $\mathbb{R}^{d}$ version of Birkhoff's Theorem.

\section{EXAMPLE}

Let $\mu_{d-1}(\gamma)=\delta(|\gamma|-1) \in M_{b+}\left(\widehat{\mathbb{R}}^{d}\right)$ be the measure corresponding to a mass distributed homogeneously over $\Sigma_{d-1}$; the total mass is taken as $\omega_{d-1}$ so that the compactly supported measure $\mu_{d-1}$ is the restriction of surface measure $\sigma_{d-1}$ to $\Sigma_{d-1}$. The transform $\mu_{d-1}^{\vee}(t)$ is easily computed in terms of the approximants $\frac{1}{\tau} \chi((|\gamma|-1) / \tau)$ to $\mu_{d-1}$, where $\chi(r)=\chi_{[-1 / 2,1 / 2)}(r)$ for $r \in \mathbb{R}$. 
In fact, we have

$$
\begin{aligned}
\mu_{d-1}^{\vee}(t) & =\lim _{\tau \rightarrow 0} \int \frac{1}{\tau} \chi\left(\frac{|\gamma|-1}{\tau}\right) e^{2 \pi i t \cdot \gamma} d \gamma \\
& =\lim _{\tau \rightarrow 0} \frac{1}{\tau} \int_{1-\tau / 2}^{1+\tau / 2} \rho^{d-1}\left(\int_{\Sigma_{d-1}} e^{2 \pi i t \cdot \rho \theta} d \sigma_{d-1}(\theta)\right) d \rho \\
& =2 \pi|t|^{-((d-2) / 2)} J_{(d-2) / 2}(2 \pi|t|),
\end{aligned}
$$

since

$$
\int_{\Sigma_{d-1}} e^{2 \pi i t \cdot \rho \theta} d \sigma_{d-1}(\theta)=2 \pi(|t| \rho)^{-((d-2) / 2)} J_{(d-2) / 2}(2 \pi|t| \rho)
$$

Theorem 4.1. Given $d \geq 2$ and the measure $\mu_{d-1}$. There is $\varphi \in L_{\text {loc }}^{2}\left(\mathbb{R}^{d}\right)$ such that

$$
\forall t \in \mathbb{R}^{d}, \quad \lim _{R \rightarrow \infty} \frac{1}{|B(R)|} \int_{B(R)} \varphi(t+x) \overline{\varphi(x)} d x=\mu_{d-1}^{\vee}(t) .
$$

Proof. The result will follow from Theorem 3.3 once we construct $\left\{\mu_{n}\right\}$ and $\left\{\varphi_{n}\right\}$ as in $\S 3$ where $\left\{\varphi_{n}\right\}$ satisfies (3.4).

To this end we first cover $\Sigma_{d-1}$ in the following way. For each $n$ we choose about $n^{d-1}$ balls $B_{j, n}, j=1, \ldots, N_{n} \leq N_{d} n^{d-1}$, each having diameter bounded by $D_{d} / n$ and whose union covers $\Sigma_{d-1}$. For $d=2$, this is doable since the circle can be divided into $n$ arcs of equal length $2 \pi / n$, and, hence, $n$ balls of diameter $D_{2} / n$ can be chosen. For $d=3$, we first consider the equator and the polar great circle. We choose $n$ equispaced points on both circles. For convenience let $n$ be even so that the polar points are marked on the polar great circle. Then spin the polar great circle stopping at each marked point on the equator. At each stop we mark points on $\Sigma_{2}$ determined by the rotated points of the polar great circle. In this way we obtain $N_{n} \leq N_{3} n^{2}$ points on $\Sigma_{2}$. For any marked point $p$ on the equator we choose a ball $B(p, r)$ where the radius $r$ is large enough to include two marked points of the equator on either side of $p$. Balls of this size centered at the other marked points on $\Sigma_{2}$ yield the desired set $\left\{B_{j, n}\right\}$ (since $B(p, r)$ covers the largest area on $\Sigma_{2}$ between a block of 6 neighboring points). For $d>3$ we use this process by considering $d-1$ "great circles" $\Sigma_{d-2}$ to find the $n^{d-1}$ points and isometric balls $B_{j, n}$ centered at these points. Analytically, this can be accomplished by imbedding $\Sigma_{d-1}$ in the unit cube, covering the surface of the unit cube by $2^{d} n^{d-1}$ balls of diameter $D_{d} / n$ (equidistributed on each face of the cube), and linearly sliding each ball to the origin where it is designated $B_{j, n}$ when its center intersects $\Sigma_{d-1}$. Clearly (geometrically), these $B_{j, n}$ cover $\Sigma_{d-1}$.

We can now construct $\left\{\mu_{n}\right\}$. For each fixed $n$ we set $B_{j, n}\left(\Sigma_{d-1}\right)=B_{j, n} \cap$ $\Sigma_{d-1}, j=1, \ldots, N_{n}$; we then obtain a partition of $\Sigma_{d-1}$ by defining $O_{1, n}=$ 
$B_{1, n}\left(\Sigma_{d-1}\right)$ and

$$
O_{j, n}=B_{j, n}\left(\Sigma_{d-1}\right) \cap\left(\sum_{k=1}^{j-1} B_{k, n}\left(\Sigma_{d-1}\right)\right)^{\sim}, \quad j=2, \ldots, N_{n} .
$$

There is $K_{d}$, independent of $n$ and $j=1, \ldots, N_{n}$, such that

$$
\mu_{d-1}\left(O_{j, n}\right) \leq K_{d} / n^{d-1} \text {. }
$$

Besides the geometric proof of (4.2) we can proceed analytically as follows. Take $g=\chi_{B_{j, n}}\left(\Sigma_{d-1}\right)$ and define $g_{d}$ on $\mathbb{R}^{d}$ by setting $g_{d}(0)=0, g_{d}(\rho \theta)=g(\theta)$ for $0<\rho \leq 1$ and $\theta \in \Sigma_{d-1}$, and $g_{d}(\gamma)=0$ for $|\gamma|>1$. By the definition of $\mu_{d-1}$

$$
\int_{\Sigma_{d-1}} g(\theta) d \sigma_{d-1}(\theta)=\int g_{d}(\gamma) d \gamma
$$

and (4.2) is obtained by calculating the right-hand side of (4.3) using spherical coordinates.

Now that we have a partition $\left\{O_{j, n}: j=1, \ldots, N_{n} \leq N_{d} n^{d-1}\right\}$ of $\Sigma_{d-1}$ satisfying (4.2) and having diameters bounded by $D_{d} / n$, we choose $\omega_{j, n} \in O_{j, n}$ (when $O_{j, n} \neq \phi$ ) and define

$$
\mu_{n}=\sum_{j=1}^{N_{n}} \mu_{d-1}\left(O_{j, n}\right) \delta_{\omega_{j, n}}
$$

and

$$
\varphi_{n}(t)=\sum_{j=1}^{N_{n}} \mu_{d-1}\left(O_{j, n}\right)^{1 / 2} e^{2 \pi i t \cdot \omega_{j, n}}
$$

It remains to prove that $\lim _{n \rightarrow \infty} \mu_{n}=\mu$ in the Levy topology (so that we have (3.6)) and that (3.4) is satisfied.

The proof that $\lim _{n \rightarrow \infty} \mu_{n}=\mu$ in the Levy topology, i.e., $\sigma\left(M_{b}\left(\widehat{\mathbb{R}}^{d}\right), C_{b}\left(\widehat{\mathbb{R}}^{d}\right)\right)$, proceeds as follows. Given $g \in C_{b}\left(\widehat{\mathbb{R}}^{d}\right)$; since $g$ is uniformly continuous on $\Sigma_{d-1}$ there is $\left\{r_{n}\right\}$ decreasing to 0 so that if $\theta, \theta^{\prime} \in \Sigma_{d-1}$ have Euclidean distance less than $D_{d} / n$ then $\left|g(\theta)-g\left(\theta^{\prime}\right)\right|<r_{n}$. Therefore, for each $j$,

$$
\begin{aligned}
\int_{\Sigma_{d-1}} \chi_{0_{j, n}}(\theta) g(\theta) d \sigma_{d-1}(\theta)= & g\left(\omega_{j, n}\right) \int_{\Sigma_{d-1}} \chi_{0_{j, n}}(\theta) d \sigma_{d-1}(\theta) \\
& +\beta_{j, n} r_{n}\left|\mu_{d-1}\right|\left(0_{j, n}\right),
\end{aligned}
$$

$\left|\beta_{j, n}\right|<1$. Summing over $j$ we obtain the desired convergence since

$$
\left|\int g(\gamma) d \mu_{d-1}(\gamma)-\int g(\gamma) d \mu_{n}(\gamma)\right|<\omega_{d-1} r_{n} \text {. }
$$

To verify (3.4) we first use (4.2) to observe that

$$
\left\|\varphi_{k}\right\|_{\infty} \leq N_{d} K_{d}^{1 / 2} k^{((d-1) / 2)} \text {. }
$$


Thus, we have

$$
\begin{gathered}
\left.\frac{1}{n !} \sum_{k=2}^{n} \max \left(\left\|\varphi_{k-1}\right\|_{\infty},\left\|\varphi_{k}\right\|\right)_{\infty}\right) \max \left(\left\|\varphi_{k}\right\|_{\infty},\left\|\varphi_{k+1}\right\|_{\infty}\right) \\
\leq \frac{N_{d}^{2} K_{d}}{n !} \sum_{k=1}^{n+1} k^{d-1} \leq N_{d}^{2} K_{d} \frac{(n+1)^{d}}{n !}
\end{gathered}
$$

The right-hand side tends to 0 and so (3.4) is satisfied. Q.E.D.

Remark 4.2. The geometrical construction in Theorem 4.1 was made with (3.4) in mind. There are other methods of partitioning $\Sigma_{d-1}$ so that the results of $\S 3$ can be invoked. For example, let $d=3$ and consider the "simplex" determined by the six points $( \pm 1,0,0),(0, \pm 1,0)$, and $(0,0, \pm 1)$. The trace of the boundary of this octahedron on $\Sigma_{2}$ (with the "eraser-end of the pencil" at the origin) determines 8 subsurfaces of $\Sigma_{d-1}$ having equal areas. At the next step we make the barycentric triangulation of each face and proceed to establish 48 subsurfaces having equal areas. In this way we produce a partition growing exponentially in cardinality, but compensated for by much finer estimates than (4.2). In particular, at the $k$ th step the partition $\left\{O_{j, k}\right\}$ has the properties that $j=1, \ldots, 2^{3} 6^{k-1}$ and $\mu_{2}\left(O_{j, k}\right)=\omega_{2} /\left(2^{3} 6^{k-1}\right)$. Thus $\left\|\varphi_{k}\right\|_{\infty} \leq\left(\omega_{2} 2^{3} 6^{k-1}\right)^{1 / 2}$ and, hence, we have the estimate (for verifying (3.4)),

$$
\begin{gathered}
\frac{1}{n !} \sum_{k=2}^{n} \max \left(\left\|\varphi_{k-1}\right\|_{\infty},\left\|\varphi_{k}\right\|_{\infty}\right) \max \left(\left\|\varphi_{k}\right\|_{\infty},\left\|\varphi_{k+1}\right\|_{\infty}\right) \\
\leq \frac{\omega_{2} 2^{3}}{n !} \sum_{k=0}^{n} 6^{k} \leq \frac{8 \omega_{2}}{5 n !} 6^{n+1},
\end{gathered}
$$

which tends to 0 . For this partitioning, our verification of convergence in the Levy topology also applies since the diameters of the $O_{j, k}$ tend to 0 as $k \rightarrow \infty$.

\section{GENERALIZED HARMONIC ANALYSIS AND SPECTRUM ESTIMATION}

Definition 5.1. Given $\varphi \in L_{\mathrm{loc}}^{2}\left(\mathbb{R}^{d}\right)$ and define

$$
\forall R>0, \quad P_{\varphi, R}=\frac{1}{|B(R)|}\left(\varphi \chi_{B(R)}\right) *\left(\varphi \chi_{B(R)}\right)^{\sim}
$$

so that $P_{\varphi, R} \in L_{\text {loc }}^{1}\left(\mathbb{R}^{d}\right) \subseteq M\left(\mathbb{R}^{d}\right)$. Suppose that there is a continuous positive definite function $P_{\varphi}$ for which $\lim _{R \rightarrow \infty} P_{\varphi, R}=P_{\varphi}$ in the $\sigma\left(M\left(\mathbb{R}^{d}\right), C_{c}\left(\mathbb{R}^{d}\right)\right)$ topology. Then $P_{\varphi} \in L^{\infty}\left(\mathbb{R}^{d}\right)$ is the autocorrelation of $\varphi$ and $\widehat{P}_{\varphi}=\mu_{\varphi}$ is the power spectrum of $\varphi$.

Given the vague convergence, $\lim _{R \rightarrow \infty} P_{\varphi, R}=P_{\varphi}$, the positive definiteness of $P_{\varphi}$ can be easily verified.

Suppose the data characterizing a given signal $\varphi$ is known. In the following result, $\hat{f}$ can be thought of as a properly shaped window function so that the 
left side of (5.1) represents the power of $\varphi$ in the region supp $\hat{f}$. Formula (5.1) provides a method for computing this power in terms of the known functions $f$ and $\varphi$. In practice, then, numerical estimates of the right-hand side of (5.1) lead to a spectrum estimation algorithm.

Theorem 5.2. Given $\varphi \in L_{\text {loc }}^{2}\left(\mathbb{R}^{d}\right)$ with autocorrelation $P_{\varphi}$ and power spectrum $\mu_{\varphi}$. Assume there is an increasing function $i(R)$ on $(0, \infty)$ for which $\sup _{|x| \leq R}|\varphi(x)| \leq i(R)$ and $\lim _{R \rightarrow \infty} i(R)^{2} / R=0$. Then

(5.1) $\forall f \in C_{c}\left(\mathbb{R}^{d}\right), \quad \int|\hat{f}(\gamma)|^{2} d \mu_{\varphi}(\gamma)=\lim _{R \rightarrow \infty} \frac{1}{|B(R)|} \int_{B(R)}|f * \varphi(t)|^{2} d t$.

(A simple estimate shows that $f * \varphi \in L_{\mathrm{loc}}^{2}\left(\mathbb{R}^{d}\right)$ for $f \in C_{c}\left(\mathbb{R}^{d}\right)$ and $\varphi \in$ $L_{\mathrm{loc}}^{2}\left(\mathbb{R}^{d}\right)$.)

Proof. (a) By Bochner's theorem we have $\mu_{\varphi} \in M_{b+}\left(\widehat{\mathbb{R}}^{d}\right)$; and, by the $\sigma\left(M\left(\mathbb{R}^{d}\right), C_{c}\left(\mathbb{R}^{d}\right)\right)$ convergence $\lim _{R \rightarrow \infty} P_{\varphi, R}=P_{\varphi}$ and the Parseval relation, we have

$$
\forall f \in C_{c}\left(\mathbb{R}^{d}\right), \lim _{R \rightarrow \infty} \int \frac{1}{|B(R)|}\left|\left(\varphi \chi_{B(R)}\right)^{\curlywedge}(\gamma)\right|^{2}|\hat{f}(\gamma)|^{2} d \gamma=\int|\hat{f}(\gamma)|^{2} d \mu_{\varphi}(\gamma) .
$$

Parseval can be applied since $\overline{(f * \tilde{f})} P_{\varphi} \in L^{1}\left(\mathbb{R}^{d}\right)$.

Using Plancherel's theorem we see that the left-hand side of (5.2) is

$$
\lim _{R \rightarrow \infty} \frac{1}{|B(R)|} \int\left|f *\left(\varphi \chi_{B(R)}\right)(t)\right|^{2} d t
$$

in particular this limit exists for each $f \in C_{c}\left(\mathbb{R}^{d}\right)$. We complete the proof once we show that (5.3) equals

$$
\lim _{R \rightarrow \infty} \frac{1}{|B(R)|} \int_{B(R)}|f * \varphi(t)|^{2} d t
$$

in particular, we prove that this limit exists, noting that $f * \varphi \in L_{\text {loc }}^{2}\left(\mathbb{R}^{d}\right)$.

(b) Using the triangle inequality for $L^{2}\left(\mathbb{R}^{d}\right)$ and the generalized Minkowski 
inequality, we have the estimate

$$
\begin{aligned}
& \left|\left(\frac{1}{|B(R)|} \int\left|f *\left(\varphi \chi_{B(R)}\right)(t)\right|^{2} d t\right)^{1 / 2}-\left(\frac{1}{|B(R)|} \int\left|(f * \varphi)(t) \chi_{B(R)}(t)\right|^{2} d t\right)^{1 / 2}\right| \\
& \quad \leq \frac{1}{|B(R)|^{1 / 2}}\left(\int\left|f *\left(\varphi \chi_{B(R)}\right)(t)-(f * \varphi)(t) \chi_{B(R)}(t)\right|^{2} d t\right)^{1 / 2} \\
& \quad=\frac{1}{|B(R)|^{1 / 2}}\left(\int\left|\int f(x)\left[\left(\varphi \chi_{B(R)}\right)(t-x)-\chi_{B(R)}(t) \varphi(t-x)\right] d x\right|^{2} d t\right)^{1 / 2} \\
& \quad \leq \frac{1}{|B(R)|^{1 / 2}} \int\left(\int\left|f(x)\left[\left(\varphi \chi_{B(R)}\right)(t-x)-\chi_{B(R)}(t) \varphi(t-x)\right]\right|^{2} d t\right)^{1 / 2} d x \\
& \quad=\frac{1}{|B(R)|^{1 / 2}} \int_{\operatorname{supp} f}|f(x)|\left[\int\left|\left(\varphi \chi_{B(R)}\right)(t-x)-\chi_{B(R)}(t) \varphi(t-x)\right|^{2} d t\right]^{1 / 2} d x,
\end{aligned}
$$

for each $f \in C_{c}\left(\mathbb{R}^{d}\right)$. Consequently, the proof of the result is complete once we show that the right-hand side of (5.5) tends to 0 as $R \rightarrow \infty$.

(c) Let $\varphi(R, t-x)=\left(\varphi \chi_{B(R)}\right)(t-x)-\chi_{B(R)}(t) \phi(t-x)$. Then $\varphi(R, t-x)$ is 0 if $t \in B(R)$ and $t \in x+B(R)$ or if $t \notin B(R)$ and $t-x \notin B(R)$. Also, $\varphi(R, t-x)=\varphi(t-x)$ if $t \notin B(R)$ and $t \in x+B(R)$, and $\varphi(R, t-x)=$ $-\phi(t-x)$ if $t \in B(R)$ and $t-x \notin B(R)$. Thus, the right-hand side of (5.5) is (5.6)

$$
\frac{1}{|B(R)|^{1 / 2}} \int_{\operatorname{supp} f}|f(x)|\left(\int_{X_{x, R}}|\varphi(t-x)|^{2} d t+\int_{Y_{x, R}}|\varphi(t-x)|^{2} d t\right)^{1 / 2} d x,
$$

where $X_{x, R}=\{t: t \notin B(R)$ and $t \in x+B(R)\}$ and $Y_{x, R}=\{t: t \in B(R)$ and $t \notin x+B(R)\}$.

If $R_{f}=\sup \{|x|: x \in \operatorname{supp} f\}$, then straightforward calculations show that $X_{x, R} \subseteq B\left(R+R_{f}\right) \backslash B(R)$ and $Y_{x, R} \subseteq B(R) \backslash B\left(R-R_{f}\right)$ for $x \in \operatorname{supp} f$ and large $R$. Consequently, we can make the estimate

$$
\begin{gathered}
\sup _{x \in \operatorname{supp} f}\left(\int_{X_{x, r}}|\varphi(t-x)|^{2} d t+\int_{Y_{x, R}}|\varphi(t-x)|^{2} d t\right)^{1 / 2} \\
\leq \sup _{x \in \operatorname{supp} f}\left(\left(\left|B\left(R+R_{f}\right)\right|-|B(R)|\right) \sup _{t \in B\left(R+R_{f}\right) \backslash B(R)}|\varphi(t-x)|^{2}\right. \\
\left.+\left(|B(R)|-\left|B\left(R-R_{f}\right)\right|\right) \sup _{t \in B(R) \backslash B\left(R-R_{f}\right)}|\varphi(t-x)|^{2}\right)^{1 / 2} \\
\leq a(\varphi, R, f)\left(\left|B\left(R+R_{f}\right)\right|-\left|B\left(R-R_{f}\right)\right|\right)^{1 / 2},
\end{gathered}
$$

where

$$
a(\varphi, R, f)=\sup _{x \in \operatorname{supp} f}\left(\sup _{t \in B\left(R+R_{f}\right) \backslash B(R)}|\varphi(t-x)|^{2}+\sup _{t \in B(R) \backslash B\left(R-R_{f}\right)}|\varphi(t-x)|^{2}\right)^{1 / 2} .
$$


By the mean value theorem we have the estimate,

$$
\left(\frac{\left|B\left(R+R_{f}\right)\right|-\left|B\left(R-R_{f}\right)\right|}{|B(R)|}\right)^{1 / 2} \leq\left(\frac{2 d R_{f}}{R}\right)^{1 / 2}\left(\frac{R+R_{f}}{R}\right)^{(d-1) / 2}
$$

Combining (5.6), (5.7), and (5.8), we see that the right-hand side of (5.5) is bounded by

$$
|\operatorname{supp} f|\|f\|_{\infty}\left(\frac{2 d R_{f}}{R}\right)^{1 / 2}\left(\frac{R+R_{f}}{R}\right)^{(d-1) / 2} a(\varphi, R, f)
$$

and this will tend to 0 , thus completing the proof, once we show $a(\varphi, R, f) / R^{1 / 2}$ tends to 0 as $R \rightarrow \infty$.

If $t \in B\left(R+R_{f}\right) \backslash B(R)$ and $x \in \operatorname{supp} f$ then $|t-x| \leq R+2 R_{f}$, and if $t \in B(R) \backslash B\left(R-R_{f}\right)$ and $x \in \operatorname{supp} f$ then $|t-x| \leq R+R_{f}$. Therefore, $a(\varphi, R, f) \leq \sqrt{2} i\left(R+2 R_{f}\right)$, and so

$$
\frac{a(\varphi, R, f)}{R^{1 / 2}} \leq \frac{\sqrt{2} i\left(R+2 R_{f}\right)}{\left(R+2 R_{f}\right)^{1 / 2}}\left(\frac{R+2 R_{f}}{R}\right)^{1 / 2}
$$

which tends to 0 by hypothesis. Q.E.D.

Corollary 5.3. Given the hypotheses of Theorem 5.2. If $f \in L^{p}\left(\mathbb{R}^{d}\right)$ and $\varphi \in$ $L^{p^{\prime}}\left(\mathbb{R}^{d}\right), 1 \leq p<\infty$, then the tempered distribution $\hat{f}$ is a well-defined element of $L_{\mu_{\varphi}}^{2}\left(\widehat{\mathbb{R}}^{d}\right)$ and

$$
\|\hat{f}\|_{2, \mu_{\varphi}} \leq\|f\|_{p}\|\varphi\|_{p^{\prime}} .
$$

Proof. If $f \in C_{c}\left(\mathbb{R}^{d}\right)$ then (5.9) is a consequence of (5.1) and Hölder's inequality. (5.9) extends to all of $L^{p}\left(\mathbb{R}^{d}\right)$ since $\overline{C_{c}\left(\mathbb{R}^{d}\right)}=L^{p}\left(\mathbb{R}^{d}\right), 1 \leq p<\infty$; as such, (5.9) simultaneously defines $\hat{f}$ and gives a quantitative norm bound (of course, $\hat{f}$ is known to exist as a tempered distribution since the elements of $L^{p}\left(\mathbb{R}^{d}\right)$ are tempered). Q.E.D.

Remarks 5.4. (a) One can formulate a version of Theorem 5.2 for $L^{\infty}\left(\mathbb{R}^{d}\right)$ instead of $L_{\text {loc }}^{2}\left(\mathbb{R}^{d}\right)$. In this case the autocorrelation can be defined in terms of the weak $* \sigma\left(L^{\infty}\left(\mathbb{R}^{d}\right), L^{1}\left(\mathbb{R}^{d}\right)\right)$ topology. As such, the analogue of Theorem 5.2 does not require any hypothesis involving $i(R)$; and the proof is much simpler since part (c) (of the proof) can be replaced by a simple estimate and application of Lebesgue dominated convergence, cf., [Me; B, pp. 89-92].

(b) For $\varphi \in L_{\text {loc }}^{2}\left(\mathbb{R}^{d}\right)$, the positive definite approximant $P_{\varphi, R}$ of $\S 5$ should be compared with the approximant

$$
Q_{\varphi, R}(t)=\frac{1}{|B(R)|} \int_{B(R)} \varphi(t+x) \overline{\varphi(x)} d x
$$


of $\S 3$. First, each $P_{\varphi, R}$ and $Q_{\varphi, R}, R>0$, is continuous, and the bounds

$$
\left|P_{\varphi, R}(t)\right| \leq \frac{1}{|B(R)|} \int_{B(R)}|\varphi(x)|^{2} d x
$$

and

$$
\left|Q_{\varphi, R}(t)\right| \leq \frac{1}{|B(R)|}\left(\int_{B(R)}|\varphi(x)|^{2} d x\right)^{1 / 2}\left(\int_{B(R)}\left|\tau_{-t} \varphi(x)\right|^{2} d x\right)^{1 / 2}
$$

are valid for all $t \in \mathbb{R}^{d}$. Second, if $\varphi$ satisfies the growth condition (in terms of $i(R))$ given in Theorem 5.2 then $\left\{P_{\varphi, R}: R>0\right\}$ and $\left\{Q_{\varphi, R}: R>0\right\}$ have the expected similar behavior at infinity. More precisely, we can show that if $\lim _{R \rightarrow \infty} P_{\varphi, R}=P_{\varphi}\left(\right.$ resp. $\left.\lim _{R \rightarrow \infty} Q_{\varphi, R}=P_{\varphi}\right)$ in the $\sigma\left(M\left(\mathbb{R}^{d}\right), C_{c}\left(\mathbb{R}^{d}\right)\right)$ topology then

$$
\lim _{R \rightarrow \infty} Q_{\varphi, R}=P_{\varphi} \quad\left(\text { resp. } \lim _{R \rightarrow \infty} P_{\varphi, R}=P_{\varphi}\right)
$$

in the $\sigma\left(M\left(\mathbb{R}^{d}\right), C_{c}\left(\mathbb{R}^{d}\right)\right)$ topology, and, in either case, $P_{\varphi}$ is positive definite. There is a corresponding pointwise result.

\section{AN ELEMENTARY RESTRICTION THEOREM}

We begin with a straightforward Fourier transform weighted norm inequality having a measure weight.

Proposition 6.1. Given $\mu \in M_{b+}\left(\widehat{\mathbb{R}}^{d}\right)$ for which $\mu^{\vee}=P \in L^{p^{\prime}}\left(\mathbb{R}^{d}\right), p \in[1, \infty]$. Then

$$
\begin{aligned}
& \forall f \in L^{1}\left(\mathbb{R}^{d}\right) \cap L^{p}\left(\mathbb{R}^{d}\right), \\
& \|\hat{f}\|_{2, \mu} \leq\|P\|_{p^{\prime}}^{1 / 2}\left(\|f\|_{1}\|f\|_{p}\right)^{1 / 2} \leq\left(\frac{1}{2}\|P\|_{p^{\prime}}^{1 / 2}\right)\left(\|f\|_{1}+\|f\|_{p}\right),
\end{aligned}
$$

where $\left(L^{1}\left(\mathbb{R}^{d}\right) \cap L^{p}\left(\mathbb{R}^{d}\right),\|\cdots\|_{1}+\|\cdots\|_{p}\right)$ is a Banach space.

Proof. The Parseval relation,

$$
\forall f \in L^{1}\left(\mathbb{R}^{d}\right), \quad \int|\hat{f}(\gamma)|^{2} d \mu(\gamma)=\int \overline{f * \tilde{f}(t)} P(t) d t,
$$

is valid since $\overline{f * \tilde{f}(t)} P(t) \in L^{1}\left(\mathbb{R}^{d}\right)$. The right-hand side of (6.2) is

$$
\int \overline{f(x)}\left[\int f(x-t) P(t) d t\right] d x \leq \int|f(x)|\|f\|_{p}\|P\|_{p^{\prime}} d x .
$$

This and the arithmetic-geometric means inequality yield the result. Q.E.D.

The following is our elementary restriction theorem with explicit norm constant.

Proposition 6.2. Given $d \geq 2$ and $1 \leq p<2 d /(d+1)$. Then $\left\|\mu_{d-1}^{\vee}\right\|_{p^{\prime}}<\infty$ and, for each $f \in L^{1}\left(\mathbb{R}^{d}\right) \cap L^{p}\left(\mathbb{R}^{d}\right)$,

$$
\left(\int_{\Sigma_{d-1}}|\hat{f}(\theta)|^{2} d \sigma_{d-1}(\theta)\right)^{1 / 2} \leq\left(\frac{1}{2}\left\|\mu_{d-1}^{\vee}\right\|_{p^{\prime}}^{1 / 2}\right)\left(\|f\|_{1}+\|f\|_{p}\right) .
$$


Proof. Using (4.1) we first show that $\mu_{d-1}^{\vee} \in L^{p^{\prime}}\left(\mathbb{R}^{d}\right)$ for $1 \leq p<2 d /(d+1)$. Not only does $J_{\nu}(s)=O\left(s^{-1 / 2}\right), s \rightarrow \infty$, e.g., Lemma 3.1, but, also, $J_{\nu}(s) \sim$ $s^{\nu} /\left[2^{\nu} \Gamma(\nu+1)\right], s \rightarrow 0, \nu>-1$. Thus, we can estimate

$$
\left\|\mu_{d-1}^{\vee}\right\|_{p^{\prime}}^{p^{\prime}}=\omega_{d-1}(2 \pi)^{p^{\prime}}\left[\int_{0}^{1} \cdots+\int_{1}^{\infty} r^{d-1} r^{-((d-2) / 2) p^{\prime}}\left|J_{(d-2) / 2}(2 \pi r)\right|^{p^{\prime}} d r\right]
$$

as follows. The first integral is finite (for any $p>1$ ) by the stated asymptotic property of $J_{\nu}$. The second integral is finite if $d-\left(d p^{\prime} / 2\right)+\left(p^{\prime} / 2\right)<0$ and this follows if $1<p<2 d /(d+1)$ and $d \geq 2$. The case $p=1$ must be treated separately but is trivial.

The result follows from Proposition 6.1. Q.E.D.

Remark 6.3. (a) In light of Theorem 4.1, the surface measure $\mu_{d-1}$ is the power spectrum of a signal $\varphi$, e.g., Definition 5.1 and Remark 5.4b. Since the norm constant in Proposition 6.2 is explicit and computable by (4.1), we see that (6.3) provides a means of estimating an upper bound for the power of $\varphi$ in the region $\operatorname{supp} \hat{f}$, even though we do not have precise knowledge of $\varphi$ itself.

(b) In Proposition 6.1 where $\mu \in M_{b+}\left(\widehat{\mathbb{R}}^{d}\right)$ is given, if $p=1$ then $P$ is always an element of $L^{\infty}\left(\mathbb{R}^{d}\right)$, and if $p=2$ then $P \in L^{2}\left(\mathbb{R}^{d}\right)$ so that $\mu \in$ $L^{1}\left(\widehat{\mathbb{R}}^{d}\right) \cap L^{2}\left(\widehat{\mathbb{R}}^{d}\right)$. In the case $\mu=\mu_{d-1}$ it is clear that $p=\infty$ cannot be used in Proposition 6.1 since $\mu_{d-1}^{\vee} \notin L^{1}\left(\mathbb{R}^{d}\right)$.

\section{ACKNOWLEDGMENT}

I would like to acknowledge important observations about the contents of this paper by Sadahiro Saeki, David Walnut, and Elmar Winkelnkemper, as well as an overall discussion with Ward Evans, Tom Harrison, Christopher Heil, and Rodney Kerby. Also, I would like to thank the referee for several valuable comments which I have incorporated into the paper.

Added in proof. Theorem 3.3 is valid for all positive bounded measures. This was observed by my student, Rodney Kerby, by making a proper choice of $\left\{R_{n}\right\}$ to satisfy condition (3.4).

\section{REFERENCES}

[Ba] J. Bass, Fonctions de corrélation fonctions pseudo-aléatoires et applications, Masson, Paris, 1984.

[B] J. Benedetto, Spectral synthesis, Academic Press, New York, 1975.

$[\mathrm{BH}]$ J. Benedetto and H. Heinig, Fourier transform inequalities with measure weights, Adv. in Math. (to appear).

[Be] J.-P. Bertrandias, Espaces de fonctions continues et bornées en moyenne asymptotique d'ordre $p$, Mém. Soc. Math. France 5 (1966).

[Bo] N. Bourbaki, Intégration, Livre VI, Hermann, Paris, 1952.

[CD] Y.-C. Chang and K. Davis, Lectures on Bochner-Riesz means, London Math. Soc. Lecture Note, Ser. 114, Cambridge Univ. Press, 1987. 
[Ma] P. Malliavin, Intégration et probabilités, analyse de Fourier et analyse spectrale, Masson, Paris, 1982.

[Me] Y. Meyer, Le spectre de Wiener, Studia Math. 27 (1966), 189-201.

[S] E. Stein, Oscillatory integrals in Fourier analysis, Beijing Lectures in Harmonic Analysis, Ann. of Math. Stud., no. 112, Princeton Univ. Press, 1986.

[T] P. Tomas, Restriction theorems for the Fourier transform in harmonic analysis in Euclidean spaces, Proc. Sympos. Pure Math., vol. 35, Part 1, Amer. Math. Soc., Providence, R. I., 1979, pp. 111-114.

[W] N. Wiener, Collected works, Vol. II, P. Masani, Ed., The MIT Press, 1979.

[WW] N. Wiener and A. Wintner, On singular distributions, J. Math. Phys. 17 (1939), 233-246 (Collected Works, Vol. II, P. Masani, Ed.).

Prometheus, INC., NewPort, Rhode Island 02840 20742

Current address: Department of Mathematics, University of Maryland, College Park, Maryland 